\title{
Autocrine Growth Hormone (GH)-Mediated Triptolide Resistance Overcame by Metformin Co-Treatment in MDA-MB231 Breast Cancer Cells Through ER Stress Pathway ${ }^{\dagger}$
}

\author{
Amani Abdulmunem, Pınar Obakan-Yerlikaya, Elif-Damla Arisan and Ajda Coker-Gurkan * \\ Department of Molecular Biology and Genetics, Istanbul Kultur University, Atakoy Campus, 34156 Istanbul, \\ Turkey; ghnoaalshikhly@gmail.com (A.A.); p.obakan@iku.edu.tr (P.O.-Y.); d.arisan@iku.edu.tr (E.-D.A.) \\ * Correspondence: a.coker@iku.edu.tr; Tel.: +90-212-498-4565 \\ + Presented at the 3rd International conference on Natural Products for Cancer Prevention and Therapy, \\ Kayseri, Turkey, 18-20 December 2019.
}

Published: 25 December 2019

\begin{abstract}
Breast cancer is the most common cancer in women worldwide and the second most common cancer overall. Autocrine growth hormone (GH) expression induced cell proliferation, growth, invasion-metastasis in vitro and in vivo breast cancer models. Moreover, forced GH signaling acts as a drug resistance profile in breast cancer cell lines against chemotherapeutic drugs such as tamoxifen, mitomycin C, doxorubicin and curcumin. Triptolide, an active plant extract from Tripterygium wilfordii, has been shown to induce apoptotic cell death in various cancer cells such a prostate, colon, breast cancer. Metformin, a common therapeutic agent for type II Diabetes mellitus, has been shown to induce autophagy, endoplasmic reticulum (ER) stress and apoptotic cell death in cancer cells. Our aim is to demonstrate the potential effect of metformin on triptolide-mediated drug resistance in autocrine GH expressing MDA-MB-231 breast cancer cells through Endoplasmic reticulum (ER) stress. Autocrine GH-mediated triptolide $(20 \mathrm{nM})$ resistance overcame by metformin (2 mM) co-teatment in MDA-MB231 breast cancer cells through accelerating cell viability loss, growth inhibition compared to alone triptolide treatment. Combined treatment increased apoptotic cell death via CHOP activation, IRE1 $\alpha$ upregulation. Consequently, we suggest that triptolide can be more effective with metformin combination in MDA-MB-231 GH+ drug resistant breast cancer cells.
\end{abstract}

Keywords: breast cancer; autocrine growth hormone; triptolide; metformin

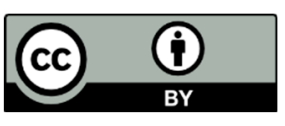

(C) 2019 by the authors. Licensee MDPI, Basel, Switzerland. This article is an open access article distributed under the terms and conditions of the Creative Commons Attribution (CC BY) license (http://creativecommons.org/licenses/by/4.0/). 